\title{
Perfil informacional de uma população jovem a respeito da AIDS e suas consequências
}

\author{
Informational profile of a young population regarding AIDS and its consequences
}

Perfil informativo de una población joven sobre el SIDA y sus consecuencias

Francisca Carolaine Araújo Costa ${ }^{1}$, Fabiane Veloso Soares², Pedro Rauel Cândido Domingos ${ }^{2 *}$.

\section{RESUMO}

Objetivo: Avaliar o conhecimento de jovens (18 a 30 anos) sobre a AIDS e suas consequências. Métodos: Foi realizado estudo transversal descritivo, com aplicação de questionário entre maio e setembro de 2018, com perguntas objetivas a respeito de conhecimentos gerais sobre HIVIAIDS. A população estudada era residente de uma capital do Norte do Brasil e precisava ter entre 18 a 30 anos. O estudo foi aprovado por Comitê de Ética em Pesquisa. Resultados: Foram realizadas 200 entrevistas, destas $59 \%$ do sexo feminino e $41 \%$ masculino. Quanto à doença, $90 \%$ dos entrevistados sabe o que AIDS, mas apenas $40 \%$ conseguiu associar a doença ao vírus. Referente à aquisição da doença, $75 \%$ associaram ao contato sexual, $88 \%$ aos materiais perfuro-cortantes e $66 \%$ acreditam que a transmissão é por contato direto. Em relação ao diagnóstico da doença, 56\% não sabem quando deve procurar ajuda médica e $15 \%$ diz que a procura por ajuda se deve a partir do surgimento dos sintomas. Conclusão: Uma parcela da população desconhece noções básicas sobre HIVIAIDS o que pode ser relacionado ao aumento de casos no país. Campanhas que utilizem meios de comunicação mais adequados aos jovens podem fomentar mudanças marcantes na prevenção ao HIV.

Palavras-chave: HIV, Saúde pública, Perfil epidemiológico, AIDS.

\begin{abstract}
Objective: To evaluate the knowledge of young people (18 to 30 years) about AIDS and its consequences. Methods: Cross-sectional study was conducted, with the application of a questionnaire between May and September 2018, with objective questions regarding general knowledge about HIV/AIDS. The population studied was a resident of a northern capital of Brazil and had to be between 18 and 30 years. The study was approved by the Research Ethics Committee. Results: 200 interviews were conducted, of which $59 \%$ were female and $41 \%$ male. As for the disease, $90 \%$ of respondents know what AIDS is, but only $40 \%$ have managed to associate a disease with the virus. Regarding the acquisition of the disease, $75 \%$ associated it with sexual contact, $88 \%$ to sharps and $66 \%$ believe that transmission is by direct contact. Regarding the diagnosis, $56 \%$ do not know when to seek medical help and $15 \%$ say that the search for help must start from the appearance of symptoms. Conclusion: A portion of the population is unaware of the basics of HIV/AIDS, which can be related to the increase in cases in the country. More campaigns using appropriate media for young people can promote significant changes in HIV prevention.
\end{abstract}

Key words: HIV, Public health, Epidemiological profile, AIDS.

${ }^{1}$ Faculdade Estácio de Manaus, Manaus - Amazonas.

${ }^{2}$ Centro Universitário do Norte (UNINORTE), Manaus - Amazonas. *E-mail: pedrorauel@gmail.com

SUBMETIDO EM: 2/2020

ACEITO EM: 3/2020

PUBLICADO EM: 4/2020

REAS/EJCH | Vol.Sup.n.47 | e3173 | DOI: https://doi.org/10.25248/reas.e3173.2020 Página 1 de 8 


\section{RESUMEN}

Objetivo: Evaluar el conocimiento de los jóvenes (18 a 30 años) sobre el SIDA y sus consecuencias. Métodos: Se realizó un estudio transversal, con la aplicación de un cuestionario entre mayo y septiembre de 2018, con preguntas objetivas de conocimiento general sobre VIH/SIDA. La población estudiada era residente de una capital en el norte de Brasil y necesitaba tener entre 18 y 30 años. El estudio fue aprobado por Comité de Ética en Investigación. Resultados: 200 entrevistas, 59\% de mujeres y $41 \%$ hombres. En cuanto a la enfermedad, $90 \%$ de los encuestados sabe lo SIDA, pero sólo $40 \%$ logró asociar el virus de la enfermedad. Relacionado con la adquisición de la enfermedad, $75 \%$ asocia con el contacto sexual, $88 \%$ a objetos punzantes y $66 \%$ creen que la transmisión es por contacto directo. En el diagnóstico, $56 \%$ no sabe cuándo buscar ayuda médica y $15 \%$ dice que la demanda de ayuda se debe a la aparición de los síntomas. Conclusión: Parte de la población desconoce los conceptos básicos del VIH/SIDA, que pueden estar relacionados con el aumento de casos en el país. Más campañas utilizando los medios adecuados para que los jóvenes pueden promover cambios significativos en la prevención del VIH.

Palabras clave: VIH, Salud pública, Perfil epidemiológico, SIDA.

\section{INTRODUÇÃO}

A Síndrome da Imunodeficiência Adquirida (Acquired Immuno deficiency Syndrome - AIDS) corresponde a um grupo de sinais e sintomas que surgem em estágio mais avançado da infecção pelo Vírus da Imunodeficiência Humana, conhecido mundialmente apenas por HIV (Human immuno deficiency virus) (BRASIL, 2006). A depressão imunológica, que afeta principalmente o número de células $\mathrm{T}_{\mathrm{C}} \mathrm{CD}^{+}$, provocada pela infecção do HIV torna o organismo hospedeiro mais vulnerável a infecções oportunistas, neoplasias secundárias e doenças neurológicas (LAZZAROTTO AR, et al., 2010; COSTA DCB, et al., 2011).

A transmissão do HIV ocorre através do contato sexual sem uso de preservativo (Infecções Sexualmente Transmissíveis - IST), contato com sangue de indivíduos contaminados e, ainda, transmissão vertical de mães infectadas para fetos ou recém-nascidos (FIOCRUZ, 2014).

Os sintomas iniciais da infecção podem ser muito semelhantes aos de outras viroses (febre e mal-estar), apesar de ser assintomática na maioria das vezes. Com a depleção do sistema imunológico a sintomatologia característica da AIDS é evidenciada, podendo surgir febre, diarreia, sudorese noturna e emagrecimento (BRASIL, 2018). Além disso, existe uma possibilidade da ocorrência de infecções oportunistas como toxoplasmose cerebral, criptococose extrapulmonar e tuberculose pulmonar (RODRIGUES-JÚNIOR AL e DE CASTILHO EA, 2010).

Apesar de inúmeras campanhas de conscientização sobre a prevenção e o diagnóstico precoce dessa virose, ela ainda é considerada um problema de saúde pública mundial, sendo estimado que cerca de 37,9 milhões de pessoas viviam com HIV em 2018, com estimativa de óbitos de 770.000 no mesmo ano (WHO, 2018). No Brasil, considerando o período de 1980 até junho de 2019, foram notificados 966.058 casos de HIV e 338.905 óbitos relacionados a essa infecção, mas os dados em relação aos casos podem não representar a realidade, visto que a notificação compulsória para o HIV começou em 2014.

O maior número de casos no Brasil se concentra nas regiões Sudeste e Sul, respectivamente, mas das cinco maiores taxas de detecção (por 100.000 hab.) observadas para as capitais brasileiras, três são capitais de estados da região norte, tendência observada a partir de 2015. A maior taxa de detecção de HIV em 2018 foi observada em homens de 25 aos 29 anos (50,8 / 100.000 hab.), sendo observada tendência de aumento de casos também em jovens desse gênero de 15 aos 24 anos, os quais praticamente dobraram suas taxas de infecção por HIV em 10 anos, saindo de 22,1 em 2008 para 41,8 / 100.000 hab. em 2018 (BRASIL, 2019),

Apesar do grande investimento público em trabalhos de prevenção e conscientização da população, de haver muita informação disponível e de fácil acesso em diversos tipos de mídia sobre formas de 
transmissão, riscos da doença, grupos de risco e principalmente sobre formas de prevenção, a faixa etária que se espera maior acesso a informação é aquela que mais contribui com o aumento de casos na atualidade (MONTEIRO RLM e MONTEIRO DLM, 2005).

A mudança de perfil dos jovens e a precocidade com a qual estão ocorrendo os relacionamentos com envolvimento sexual exigem novas formas de orientação sexual, principalmente em relação às IST (BORGES ALV e SCHOR N, 2005). Na atualidade existe uma diversidade de formas de mídia que podem ser exploradas para campanhas contra infecções sexualmente transmissíveis e o presente trabalho vem de encontro com a necessidade de se conhecer melhor os jovens contemporâneos, quanto ao conhecimento que eles têm sobre a $A I D S$, doença de importância epidemiológica elevada. Estudos desse tipo podem fomentar campanhas direcionadas ao público identificado como mais vulnerável e diminuir o número crescente de casos nessa faixa etária mais jovem.

Diante do exposto, o presente estudo teve como objetivo avaliar o conhecimento de jovens de 18 a 30 anos, residentes em uma capital localizada na região Norte do Brasil, sobre AIDS/HIV, identificando variáveis significativas, entre os dados coletados, que possam auxiliar na identificação de um perfil informacional dessa população sobre o tema. Esse perfil poderá ser utilizado na proposição de medidas de educação e conscientização direcionadas aos grupos de identificados como os de maior risco para essa infecção.

\section{MÉTODOS}

A pesquisa (aprovada pelo Comitê de Ética em Pesquisa da Faculdade Estácio do Amazonas, sob o oㅡ parecer 2.297.990), consistiu em um estudo transversal, com coleta de dados direta por meio de questionário padrão, com perguntas sobre prevenção, identificação, diagnóstico e prognóstico de indivíduos infectados pelo HIV, além de conhecimentos básicos sobre AIDS. A aplicação dos questionários foi realizada entre os meses de maio e setembro de 2018 a todas as pessoas que demonstraram interesse em participar e que assinaram o Termo de Consentimento Livre e Esclarecido (TCLE), conforme Resolução 196/96 da Comissão Nacional de Ética em Pesquisa (CONEP).

Os questionários foram aplicados em diferentes terminais rodoviários localizados em pontos estratégicos, incluindo a região central, de uma capital localizada na região Norte do Brasil. A escolha dos locais levou em consideração os terminais com rotas de ônibus com grande circulação de pessoas e que cobriam uma extensa área da capital, o que possibilitou a abordagem de jovens de diferentes regiões da cidade.

Os critérios de inclusão no estudo foram apresentar idade entre 18 e 30 anos, ter aceitado as condições da pesquisa e assinado o TCLE, residir no município a pelo menos 10 anos e ter respondido todas as questões propostas no questionário, e os critérios de exclusão: ser profissional da saúde com formação superior e ter formação superior incompleta (mais de dois períodos cursados) na área da saúde.

A partir das frequências obtidas para cada resposta, foram calculadas taxas de prevalência, considerando como variáveis, para fins comparativos, aspectos pessoais/sociais e instrucionais, a fim de se estabelecer perfis por sexo e por faixa etária.

\section{RESULTADOS E DISCUSSÃO}

Ao final do estudo foram realizadas 200 entrevistas que se encaixavam nos critérios propostos, onde foi verificada uma maior participação das mulheres $(59 \%)$ em relação aos homens (41\%). A idade média dos entrevistados foi de $22 \pm 3,96$ anos, a maioria deles possuíam ensino médio completo (45\%) e renda média de $R \$ 1.196,00$.

Quando questionados sobre vida/histórico sexual foi verificado que a maioria dos entrevistados manteve a primeira relação sexual por volta dos 15 anos (20\%) e que $25 \%$ afirmaram não ter usado camisinha na primeira relação (Tabela 1). O início da vida sexual tem acontecido cada vez mais cedo no Brasil (HUGO 
TDO, et al., 2011), mas, embora tenha ocorrido um aumento no uso de preservativo na primeira relação sexual ao longo dos anos, a proporção desse uso tem relação direta com o nível de escolaridade, sendo os mais escolarizados aqueles que dedicam maior cuidado na prática sexual (PAIVA V, 2008).

A imaturidade no início da atividade sexual, principalmente, mas não exclusivamente, em decorrência da pouca idade, pode ser associada a essa elevada taxa de indivíduos que não utilizam preservativo na primeira relação sexual.

Tabela 1 - Comportamento sexual, acesso a veículos de informação/campanhas sobre HIV/AIDS e principais origens/veículos da informação descritos nas entrevistas.

\begin{tabular}{|c|c|c|c|}
\hline Comportamento sexual & Sim & Não & Não soube responder \\
\hline Uso do preservativo na $1^{\circ}$ relação sexual & $72 \%$ & $25 \%$ & $3 \%$ \\
\hline Vida sexual ativa & $80 \%$ & $17 \%$ & $3 \%$ \\
\hline Parceiro fixo & $57 \%$ & $40 \%$ & $3 \%$ \\
\hline Acesso à informação & Sim & & Não \\
\hline Acesso a veículos de informação & $94 \%$ & & $6 \%$ \\
\hline Viu alguma campanha contra AIDS & $93 \%$ & & $7 \%$ \\
\hline Considera as campanhas esclarecedoras & $85 \%$ & & $15 \%$ \\
\hline Mudou algum comportamento por causa da campanha sobre AIDS & $65 \%$ & & $35 \%$ \\
\hline Teve educação sexual na adolescência & $80 \%$ & & $20 \%$ \\
\hline \multicolumn{4}{|l|}{ Origem da informação* } \\
\hline TV & $49 \%$ & & \\
\hline Escola & $31 \%$ & & \\
\hline Unidades/profissionais de saúde & $20 \%$ & & \\
\hline Internet/ redes sociais & $11 \%$ & & \\
\hline
\end{tabular}

Legenda: * $O$ entrevistado poderia citar mais de uma origem de informação.

Fonte: Costa FCA, et al., 2020.

Quanto ao comportamento sexual dos entrevistados, verificou-se que a maioria deles tem vida sexual ativa (80\%), praticamente sem diferença entre homens e mulheres (Homens $82 \%$ e Mulheres $78 \%$ ), e $57 \%$ (Homens $47 \%$ e Mulheres $66 \%$ ) afirmaram ter parceiros fixos nessas relações. Essa diferença de comportamento sexual quanto a parceiro fixo é comum, havendo relatos de que os homens tendem a ter menos relacionamentos estáveis (parceiras fixas) quando comparados às mulheres (BARBOSA RM e KOYAMA MAH, 2008). Brasil (2019) destaca que a maior ocorrência de casos de HIV é notificada entre homens jovens, até os 30 anos. Esse dado quando relacionado à característica observada para o gênero de manter menos relacionamentos fixos pode está relacionado a uma maior participação masculina na disseminação do vírus, principalmente quando associado a comportamentos de risco.

Segundo os entrevistados, eles tiveram acesso a diferentes fontes de informações sobre HIVIAIDS durante a vida, mas apesar de $93 \%$ afirmarem ter acesso aos meios de comunicação baseados na internet, apenas $11 \%$ afirmou ter visto alguma campanha de prevenção da AIDS nesse veículo. Esse dado referente à internet evidencia a potencialidade desse veículo de informação, que vem sendo subutilizado quanto à finalidade de conscientização sobre $A I D S$, podendo ser explorado inclusive para outras patologias de importância epidemiológica. A TV foi a fonte de informação mais citada pelos entrevistados (49\%), seguida pela escola (31\%), unidades de saúde $(20 \%)$ e internet/redes sociais $(11 \%)$. Em um estudo semelhante, Natividade JC e Camargo BV (2011) verificaram que a internet é um dos meios com menos campanhas de conscientização e prevenção da AIDS.

A maioria dos entrevistados (85\%) considerou como esclarecedora as campanhas que viram/ouviram ou participaram e $65 \%$ deles afirmaram mudar algum comportamento considerado de risco por influência dessas campanhas, principalmente em relação ao uso do preservativo. Quando questionados sobre a educação sexual durante a adolescência, $20 \%$ dos entrevistados afirmaram que não tiveram nenhum tipo de educação sexual na faixa etária de 10-20 anos. A educação sexual nas escolas é de suma importância, pois a partir dela os jovens e adolescentes são orientados, informados e esclarecidos quanto às formas de 
contracepção e de prevenção de doenças sexualmente transmissíveis, especialmente AIDS (CARVALHO LG e DA SILVA LL, 2017). No âmbito familiar, mesmo que a educação sexual não seja um assunto "aberto", é dever da família participar nessa orientação, principalmente quanto ao ensino de conceitos básicos e de comportamentos mais adequados para resguardar a saúde (JARDIM DP e BRETAS JRS, 2006).

A partir dos 10 anos de idade os brasileiros já utilizam a internet, para os mais diversos fins, desde acadêmicos aos recreativos. A grande maioria (95\%) dos jovens, com idade entre os 18 e 24 anos, apresentam uma elevada participação em redes sociais, o que evidencia o quanto esse meio de comunicação tem potencial como ferramenta na prevenção de doenças (DAMASCENO EB, et al., 2019). A utilização de redes sociais para divulgar materiais com objetivo de orientação e conscientização quanto a medidas preventivas para a AIDS, assim como para outras doenças, pode ser o diferencial que faltava para que os órgãos de saúde alcancem o público jovem com mais eficiência, principalmente diante da emergência que é a prevenção de ISTs nessa faixa etária, na qual a evolução do número de casos foi mais marcante nos últimos anos (BRASIL, 2019).

Características básicas sobre a infecção que promove a AIDS também são desconhecidas pela maioria, fato verificado pela incapacidade de $60 \%$ dos entrevistados em associar a infecção pelo vírus da HIV com a consequente AIDS naqueles não tratados. Esse dado chamou a atenção pelo fato de $97 \%$ deles terem afirmado saber o que é a AIDS (Tabela 2).

Tabela 2 - Principais formas de transmissão/aquisição do HIV e domínio de conhecimentos básicos sobre AIDS (doença, diagnóstico e prognóstico), segundo os entrevistados.

\begin{tabular}{lccc}
\hline Formas de transmissão & SIM & NÃO & Não soube responder \\
\hline Transmissão por contato sexual & $75 \%$ & $18 \%$ & $7 \%$ \\
Transmissão vertical & $89 \%$ & $11 \%$ & - \\
Transmissão por materiais perfuro-cortantes & $88 \%$ & $12 \%$ & - \\
Transmissão por contato direto & $34 \%$ & $66 \%$ & - \\
\hline Conhecimentos básicos sobre AIDS & SIM & NÃO \\
\hline Sabe o que é AIDS? & $97 \%$ & $3 \%$ \\
Conhece a sua causa? & $40 \%$ & $60 \%$ \\
Sabe como se prevenir? & $85 \%$ & $15 \%$ \\
Sabe quando é necessário buscar o diagnóstico? & $44 \%$ & $56 \%$ \\
Sabe quais são os sintomas da AIDS? & $67 \%$ & $33 \%$ \\
A AIDS tem cura? & $7 \%$ & $93 \%$ \\
O portador do vírus HIV pode ter uma vida normal? & $79 \%$ & $21 \%$ \\
Você considera essa doença perigosa? & $98 \%$ & & $2 \%$ \\
A AIDS pode matar? & $88 \%$ & & $12 \%$ \\
\hline
\end{tabular}

Fonte: Costa FCA, et al., 2020.

Ao serem questionados sobre as formas de se contrair a infecção pelo HIV, onde os entrevistados poderiam listar mais de uma forma, $75 \%$ deles associaram a infecção com o contato sexual, $88 \%$ a materiais perfuro-cortantes e $66 \%$ afirmam não ser possível a transmissão por contato direto, enquanto a transmissão vertical é considerada possível para grande maioria dos entrevistados (89\%). Santos VP, et al. (2017) em pesquisa semelhante, mas com público formado apenas por universitários, verificou que 98,3\% associam a transmissão do HIV ao contato sexual e 97,6 a materiais perfuro-cortantes, resultados superiores aos observados no presente estudo. Essa diferença entre os resultados pode ser justificada pela diferença no nível de escolaridade médio do público utilizado nas duas pesquisas.

A transmissão do vírus se dá pelo contato com secreções como sangue, esperma, sangue, secreção vaginal e leite materno, onde o vírus aparece em quantidades suficientes para causar infecção, o contato desses líquidos com o organismo pode ser por exposição sexual desprotegida, amamentação, gestação e uso de materiais perfuro-cortantes contaminados, além de transfusões sanguíneas (FIOCRUZ, 2014). A falta de informações sobre características básicas pode ser sanada na educação básica e média, não descartando a participação de familiares nessa orientação, sendo algo fundamental para o entendimento da importância da prevenção, do diagnóstico e do tratamento precoce. 
A prevenção é uma informação que poderíamos descrever como básica, sendo verificada que para $85 \%$ dos entrevistados o uso do preservativo é a principal forma de prevenção, o que destaca o conhecimento dos entrevistados sobre a característica de transmissão sexual que esse vírus apresenta. Apesar de apenas $15 \%$ dos entrevistados terem afirmado desconhecer um método de prevenção, esse dado é preocupante, visto que a maioria das campanhas educativas sobre AIDS investem muito nesse ponto. Francisco MTR, et al. (2014), em estudo feito com participantes do carnaval do Rio de Janeiro (RJ), também verificaram que a maioria apontam o uso de preservativos como a principal forma de prevenção (93\%), tendo resultado mais representativo do que no presente estudo, provavelmente, pela diferença de público entrevistado.

A forma de transmissão é amplamente divulgada em materiais de propaganda, sendo concentrados esforços nessas campanhas na evidenciação da importância do uso do preservativo na prevenção da AIDS (BRASIL, 2019). Apesar disso ainda existe uma parcela da população que desconhece a principal forma de prevenção ( $15 \%$ no presente estudo), destacando a necessidade de se discutir prevenção de uma maneira mais completa, fazendo com que o publico conheça mais as particularidades da infecção pelo HIV, possibilitando ao público fazer essas associações de causa e efeito de maneira natural.

Entrevistados que não apontaram o uso de preservativo como uma proteção contra o a infecção pelo $H I V$ podem estar associados ao grupo daqueles que afirmaram não considerar as campanhas sobre AIDS esclarecedoras, o que apontaria para a necessidade de se rever o conteúdo ou formato do material apresentado. Lima MM, et al. (2008), destaca que a falta de conhecimento sobre alguns assuntos relacionados a AIDS pode causar discriminação de certos grupos, sendo fundamental que nas campanhas sejam apresentadas as formas de contágio e as de não contágio. Nesse sentido, poderiam ser abordados em paralelo aos métodos preventivos reais, os principais erros e dúvidas associadas à transmissão do HIV.

O contato direto, por exemplo, foi apontado por menos da metade dos entrevistados (34\%), apesar da transmissão sexual ser uma forma de contato direto, uma vez que não precisa de vetores para transmissão. O que chama a atenção nesse dado é que a grande maioria reconhece a transmissão sexual como a principal forma, mas não conseguiram associar essa forma de transmissão ao contato direto com secreções genitais, apesar de contato direto ser mais relacionado à dispersão aérea de patógenos, evidenciando que a relação entre os conceitos, apesar de básica, não foi evidente para todos.

A manifestação da doença (sinais e sintomas) também foi um parâmetro que a maioria dos entrevistados mostrou desconhecimento, sendo verificado que $67 \%$ dos entrevistados não conhecem nenhum sintoma associado a infecção pelo HIV. Quanto ao prognóstico, $79 \%$ dos entrevistados responderam que uma pessoa diagnosticada com essa infecção pode ter uma vida normal, a maioria dos entrevistados (98\%) afirmou que a AIDS é uma doença perigosa e que pode levar a óbito (88\%), e $7 \%$ acreditam que a doença tem cura. Apesar de mostrarem conhecimento sobre a importância que essa infecção pode apresentar, a crença de que é uma situação curável é preocupante, uma vez que até o momento não foi desenvolvido nenhum tratamento que promova a cura para infecção pelo HIV (MONTEIRO JFA e FIGUEIREDO MAC, 2009).

O Ministério da Saúde (BRASIL, 2016), ao fazer o mesmo questionamento, verificou que $80,4 \%$ das pessoas sabem que a AIDS não tem cura, valor inferior ao observado no nosso estudo (93\%). Essa diferença em relação aos nossos resultados pode ser justificada com base no número de entrevistados e na faixa etária consultada no estudo, de 15 a 64 anos, o que pode apontar para uma vulnerabilidade alta para infecções por HIV em outras faixas etárias não exploradas no presente trabalho. A ideia por parte da população de que essa infecção tem cura pode ser a causa da falsa impressão de segurança que pode estar levando uma boa parte da população a não tomar os cuidados necessários à prevenção dessa infecção.

Quando questionados sobre o diagnóstico, $56 \%$ dos entrevistados não sabem quando deve procurar suporte médico para isso, enquanto $15 \%$ afirmaram que a procura por esse suporte deve ocorrer apenas quanto surgirem os sintomas da infecção. A procura por ajuda médica deve ser feita quando houver comportamento de risco, seja por ter relação sexual sem uso do preservativo ou acidente com material perfuro-cortante contaminado, por exemplo. O diagnóstico precoce é de suma importância para assegurar 0 
paciente de ter uma maior qualidade de vida, dada pelo imediato início do tratamento, além de se ter conhecimento de que é portador do vírus, podendo evitar a transmissão a outras pessoas (FERREIRA MPS,et al., 2001).

Diferente de outras infecções onde a sintomatologia é o principal achado que aponta para ela, o HIV é um vírus que pode passar muito tempo despercebido, e o pensamento de que apenas com o aparecimento dos sintomas a patologia se torna um risco é errada, uma vez que o infectado pode estar atuando como um disseminador do vírus. A importância do diagnóstico precoce vem sendo abordado como estratégia para um tratamento mais eficaz, e para evitar que o portador possa infectar outras pessoas, com isso o Ministério da Saúde vem tratando desse ponto como foco da campanha de conscientização da AIDS como foi no último ano (2019), estimulando as pessoas a fazerem o teste de rápido, para que possam vir a não desenvolver a doença e ter uma sobrevida maior (BRASIL, 2019).

Quando perguntados se o portador do vírus pode ter uma vida normal, $21 \%$ dos entrevistados acredita que não, embora nos últimos anos tenha-se aumentado muito o conhecimento sobre a doença $\mathrm{e}$ desenvolvido o tratamento com coquetéis, entre outras ferramentas já desenvolvidas, tudo isso proporcionando ao portador uma sobrevida maior, deixando de ser uma doença irreversível e fatal, para tornar-se uma doença crônica, passível de tratamento (REIS RK e GIR E, 2010).

\section{CONCLUSÃO}

A jovem população estudada apresenta conhecimentos básicos sobre a infecção pelo HIV e sobre a AIDS, mas ainda existe certo desconhecimento sobre informações de extrema relevância para seu controle. Fatores de risco como início precoce da atividade sexual, relacionamento sem parceiro fixo e uso de preservativos não contrastaram com a realidade nacional, mas o desconhecimento de uma parcela da população sobre formas de prevenção, sintomas, tratamento e, principalmente, sobre o momento para se procurar auxílio médico, trazem um problema em potencial que pode está relacionado ao aumento de infecções pelo HIV e dos casos de AIDS notificados na atualidade em todo mundo, principalmente entre os mais jovens. Mudanças de comportamentos de risco podem ser estimuladas a partir de campanhas planejadas especificamente para públicos mais vulneráveis, além da utilização de plataformas com base na internet apresentarem grande potencial para esse fim, apesar de terem sido descritas pelos entrevistados como subutilizadas. A utilização de campanhas e do meio de comunicação mais adequado ao acesso pelos jovens pode fomentar mudanças marcantes na prevenção e combate ao HIV.

\section{AGRADECIMENTOS}

Agradecemos a Faculdade Estácio do Amazonas, onde o presente trabalho começou como projeto de iniciação científica (PIBIC/IES Faculdade Estácio, Período: 07/2016-08/2017). E um agradecimento especial a todas as pessoas que dedicaram alguns minutos do seu dia para participar das entrevistas, sem as quais não seria possível alcançar nenhum dos resultados aqui apresentados.

\section{REFERÊNCIAS}

1 BARBOSA RM, KOYAMA MAH. Comportamento e práticas sexuais de homens e mulheres, Brasil 1998 e 2005. Revista Saúde Pública, 2008; 42(1): 21-33.

2 BORGES ALV, SCHOR N. Início da vida sexual na adolescência e relações de gênero: um estudo transversal em São Paulo, Brasil, 2002. Caderno de Saúde Pública, 2005; 21(2): 499-507.

3 BRASIL. Departamento de Vigilância, Prevenção e Controle das IST, HIV / Aids das Hepatites Virais. O que é HIV. 2018. Disponível em:< http://www.aids.gov.br/pt-br/publico-geral/o-que-e-hiv >. Acesso em: 23 abr. 2018.

4 BRASIL. Ministério da Saúde. Secretaria de Vigilância em Saúde. Departamento de Doenças de Condições Crônicas e Infecções Sexualmente Transmissíveis - DCCI. Boletim Epidemiológico de HIV e Aids. 2019. Disponível em: < http://www.aids.gov.br/pt-br/pub/2019/boletim-epidemiologico-de-hivaids-2019>. Acesso: 07 abr. 2020. 
5 BRASIL. Ministério da Saúde. Secretaria-Executiva. Secretaria de Vigilância em Saúde. Glossário temático: DST e AIDS / Ministério da Saúde, Secretaria-Executiva, Secretaria de Vigilância em Saúde. - Brasília: Editora do Ministério da Saúde, 2006: 56.

6 CARVALHO LG, DA SILVA LL. Educação Sexual na Escola. Revista Pandora Brasil, 2017; 83: 1-7.

7 COSTA DCB, et al. Oral manifestations in HIV patients in highly active antiretroviral therapy (HAART) era - What change? - An update for the clinical. Int J Dent, 2011; 10(2): 97-102.

8 DAMASCENO EB, et al. "Algo tão simples de viver e controlar, mas difícil de compartilhar e defender": HIVIAids, segredos e socialidades em uma rede social on-line. Interface (Botucatu) [online], 2019; 23(1): e180506. Disponível em:<https://doi.org/10.1590/interface.180506>. Acesso em: 02 abr. 2020.

9 FERREIRA MPS, et al. Testagem sorológica para o HIV e a importância dos Centros de Testagem e Aconselhamento (CTA) - resultados de uma pesquisa no município do Rio de Janeiro. Ciência \& Saúde Coletiva, $2001 ; 6(2): 481-490$.

10 FIOCRUZ. Sintomas, transmissão e prevenção- 2014. Disponível em: <https://www.bio.fiocruz.br/index.php/sintomas-transmissao-e-prevencao-hiv-dpp>. Acesso em: 23 mar. 2018.

11 FRANCISCO MTR, et al. Conhecimento sobre HIV/aids e a utilização do preservativo entre os participantes do carnaval. Revista Cubana de Enfermería, 2015; 30(3): 1561-2961.

12 HUGO TDO, et al. Fatores associados à idade da primeira relação sexual em jovens: estudo de base populacional. Caderno de Saúde Pública, 2011; 27(11): 2207-2214.

13 JARDIM DP, BRETAS JRS. Orientação sexual na escola: a concepção dos professores de Jandira - SP. Revista Brasileira Enfermagem, 2006; 59(2): 157-162.

14 LAZZAROTTO AR, et al. HIV / AIDS e Treinamento Concorrente: a Revisão Sistemática. Revista Brasileira de Medicina e Esporte, 2010; 16(2): 149-154.

15 LIMA MM, et al. O conhecimento da população de Viçosa, MG, sobre as formas de transmissão da Aids. Ciência e Saúde Coletiva, 2008; 13(6): 1888-2008.

16 MONTEIRO JFA, FIGUEIREDO MAC. Vivência profissional: subsídios à atuação em HIV/Aids. Paidéia (Ribeirão Preto), 2009; 19(42): 67-76.

17 MONTEIRO RLM, MONTEIRO DLM. A mídia na informação sobre saúde sexual. Revista Adolescência e Saúde, $2005 ; 2(1): 17-28$.

18 NATIVIDADE JC, CAMARGO BV. Representações sociais, conhecimento científico e fontes de informação sobre Aids. Rede de Revistas Científicas de América Latina y el Caribe, España y Portugal, 2011; 21(49): 165-174.

19 PAIVA V. Idade e uso de preservativo na iniciação sexual de adolescentes brasileiros. Revista de Saúde Pública, 2008; 42(1): 45-53.

20 REIS RK, GIR E. Convivendo com a diferença: o impacto da sorodiscordância na vida afetivo-sexual de portadores do HIV/AIDS. Rev. esc. enferm. USP, 2010; 44(3): 759-765.

21 RODRIGUES-JÚNIOR AL, DE CASTILHO EA. AIDS e doenças oportunistas transmissíveis na faixa de fronteira Brasileira. Revista da Sociedade Brasileira de Medicina Tropical, 2010; 43(5): 542-547.

22 SANTOS VP, et al. Existe relação entre o conhecimento de estudantes a respeito das formas de contágio do HIV/AIDS e suas respostas sobre a proximidade com soropositivos? Ciências em Saúde Coletiva, 2017; 22(8): 2745-2752.

23 WHO. Global Health Observatory data repository - Number of people (all ages) living with HIV. Last updated: 201909-11. Disponível em: <https://apps.who.int/gho/data/node.main.620?lang=en>. Acesso em: 07 abr. 2020. 